\title{
Um panorama da utilização dos ambientes digitais no ensino de arquitetura frente aos níveis de computabilidade: Revisão a partir dos Congressos Graphica (2005-2017) ${ }^{1}$
}

\author{
An overview of the use of digital \\ environments in the teaching of architecture \\ according to the levels of computability: \\ Review from the Graphica Congresses (2005-2017)
}

${ }^{1}$ Versão expandida do artigo selecionado nos Anais do Graphica 2019

\begin{abstract}
Tássia Borges de Vasconselos

Mestre - Doutoranda no Programa de Pós-Graduação em Arquitetura e Urbanismo do Instituto de Arquitetura e Urbanismo da Universidade de São Paulo (IAU-USP) - São Carlos, Brasil tassiav.arq@gmail.com
\end{abstract}

\section{David Sperling}

Doutor em Arquitetura e Urbanismo

Docente do Instituto de Arquitetura e Urbanismo, Universidade de São Paulo (IAU-USP) - São Carlos, Brasil sperling@sv.usp.br

\section{RESUMO}

Este artigo, versão ampliada de trabalho apresentado no Graphica 2019, apresenta resultados parciais de pesquisa em nível de doutorado acerca da apropriação de tecnologias de representação gráfica e design digital no ensino de arquitetura no contexto brasileiro. Este estudo toma como referências as sistematizações estabelecidas por Oxman (2006) sobre a interação entre o projetista e a representação de um objeto concebido com auxílio de ambiente digital, e as que realizou posteriormente Kotnik (2010) sobre os níveis de computabilidade no processo de projeto. Assim, foi estruturada uma pesquisa correlacional, que busca a sistematização do cenário a partir de dois conjuntos de dados. 0 primeiro focado em compreender aspectos inerentes aos cursos de graduação em Arquitetura e Urbanismo, e o segundo a partir da classificação de práticas didáticas selecionadas por meio de uma revisão sistemática baseada em handsearching. Apresenta como resultado dados e reflexões sobre as práticas de design digital no ensino em Arquitetura relatadas nos Congressos Graphica no período de 2005 a 2017.

Palavras-chave: Projeto digital de arquitetura; Práticas didáticas; Ensino de Arquitetura e Urbanismo no Brasil; Revisão sistemática; Mapeamento.

\section{ABSTRACT}

This paper, an expanded version of the previous study presented at Graphica 2019, shows partial results from a PhD thesis regarding to the appropriation of graphic representation technologies and digital design in the teaching of Architecture in Brazil. This study apply as references the systematizations established by Oxman (2006) about the interaction between the designer and the representation of an object created in digital environment, and by Kotnik (2010) concerning the levels of computability in the design process. Therefore, a correlational research was structured to systematize the scenario from two groups of data. The first one was focused on the aspects from the undergraduate Brazilian courses of Architecture and Urbanism, and the second was based on the classification of didactic practices selected from a systematic review based on handsearching. As results, it is presented the data and reflections from the digital design practices applied in teaching of architecture, reported at Graphica Congresses from 2005 to 2017.

Keywords: Digital Architectural Design; Didactic Practices; Architecture and Urbanism Teaching in Brazil; Systematic Review; Mapping. 


\section{INTRODUÇÃO}

Há décadas pautas relacionadas à sistematização do processo projetual nas áreas criativas foram introduzidas e debatidas por pesquisadores e professores como Broadbent (1973), Lawson (1980), e Silva (1987), dentre outros. Broadbent (1973), por exemplo, identificou um ruído em muitos estudos até então existentes sobre o processo projetual, questionando a associação recorrente ao componente da "criatividade", principalmente, por sua descrição como uma condição estática ou inata. Nesta esteira, Lawson (1980) defendeu a necessidade de desmistificação do "arquiteto gênio", a constituição de um campo de investigação sobre os processos e metodologias de projeto e sua introdução na formação de um Designer ou Arquiteto. No âmbito do ensino de arquitetura, contemplando também a prática profissional, Silva (1987) abordou a natureza complexa da ação projetual diante da necessidade de síntese de uma grande quantidade de informação advinda de diferentes áreas do conhecimento. 0 autor também indicou a necessidade da incorporação de instrumentos lógicos que deem apoio as decisões no processo de projeto.

Atualizando esse debate a partir da emergência dos diversos ambientes digitais orientados ao projeto em arquitetura e urbanismo, pretende-se expor aqui considerações sobre as potencialidades dessas tecnologias compreendendo-as como instrumentos lógicos, assim como apresentar dados e reflexões sobre as práticas de design digital no ensino em Arquitetura no Brasil relatadas nos Congressos Graphica no período de 2005 a 2017.

\subsection{A Tecnologia incorporada no processo de projeto}

0 processo de concepção de um objeto arquitetônico esteve sempre associado ao conceito de representação e consequentemente estabelecido como o resultado da interação de um designer/arquiteto com o desenho em algum suporte físico (WOODBURY, 2010). Porém, com a emergência da revolução digital surgem novas possibilidades de concepção de arquitetura utilizando-se de outros meios (KOLAREVIC, 2003).

Inicialmente é importante resgatar que a utilização do computador como ferramenta no processo de desenho e projeto não é uma novidade para arquitetura, visto que ultrapassa meio século deste seu reconhecimento e apropriação. No entanto, precisa-se compreender que esta apropriação da tecnologia no contexto da arquitetura está sendo gradual. Hatem (2005) resgata as raízes destas implementações, indicando as principais influências, as quais foram estabelecidos entre 1962 e 1972.

Celani (2003) cita William Mitchell que indicava que, no contexto norte-americano, a utilização destas tecnologias como ferramentas de desenho e projeto ocorreu a partir da década de 80 , com a popularização dos computadores pessoais tanto no ensino em faculdades de arquitetura quanto na prática de escritórios. 
Os sistemas CAD já em 1970 eram potentes o bastante para explorar funcionalmente modelagem de sólidos. As primeiras incursões do Massachusetts Institute of Technology em experimentações acerca de representação e projeto no ambiente digital já preconizavam a flexibilidade de modificação estabelecida no desenho parametrizado. No entanto, é importante destacar que diante das potencialidades dos computadores disponíveis para a grande massa dos consumidores das ferramentas na década de 80 e 90, os sistemas CAD tiveram que se adequar. Houve, então, um "deslocamento dos objetivos originais do CAD de auxiliar do processo de criação para auxiliar da representação do projeto" (CELANI, 2003 pag. 5).

Além do valor elevado dos sistemas e da existência de déficits nas implementações, existiam as preferências do usuário pelo "código" instituído do desenho bidimensional, ou seja, os projetistas "ficavam mais confortáveis projetando em 2D" (EASTMAN, et al., 2014, pag. 27). Assim, o autor indica que naquele momento foram adotados sistemas de editores de desenhos para o desenvolvimento de projeto tradicionais, estabelecidos na lógica bidimensional.

Mesmo com todas estas questões, naturais em contextos de transformações tecnológicas, diferentes barreiras vêm sendo rompidas gradualmente e novas potencialidades vêm sendo investigadas com a incorporação de outros softwares no âmbito da arquitetura. Pode-se perceber principalmente nos últimos anos, tanto na prática em escritórios quanto no ambiente de ensino, que o processo de projeto de arquitetura não vem passando ileso a esta revolução digital. E estas transformações refletem incorporações de modificações dos programas computacionais gráficos, cada vez mais potentes, conforme abordado por Botchway; Abanyie; Afram (2015).

Neste processo, somam-se ainda as possibilidades de fabricação digital, que permitem conexões diretas entre arquivos digitais e processos de materialização. Unindo o desenvolvimento projetual à possibilidade concreta de produção, estas explorações ganham maiores possibilidades de aplicação no campo da arquitetura e do urbanismo. Nesta direção, pode-se constatar no início do milênio que os discursos dos pesquisadores da área tornam-se cada vez mais otimistas, como pode ser verificado pelas posições de Kolarevic (2003) e Mitchell (2004). Oxman \& Oxman (2014) indicaram, por sua vez, que foi a partir da virada do milênio que a lógica do design thinking começou a ser utilizada de fato no ambiente digital. E, vislumbrando as possibilidades para o futuro, Woodburry (2010) apontou que as práticas de processo de projeto sofreriam mais alterações em um curto tempo do que nas últimas décadas.

\subsection{A comunicação entre o designer e o ambiente digital - aspectos conceituais sobre interação e níveis de computabilidade}

0 processo projetual na arquitetura sempre esteve intimamente conectado à representação. Sendo tão relevante à área, a ação de representar ou desenhar foi historicamente associada à própria profissão do arquiteto, e consequentemente, com o saber "projetar", como expressado por Lawson: 
A imagem arquetípica do projetista é de alguém sentado à prancheta. Mas o que fica claro é que ele exprime as suas ideias e trabalha de modo muito gráfico e visual. Realmente, seria muito difícil tornar-se um bom projetista sem desenvolver a capacidade de desenhar bem. (LAWSON, 1980, pag. 24)

No âmbito das experimentações possibilitadas pelo avanço da utilização das tecnologias digitais em processos projetuais se estabelecem novas formas de comunicação para além da transposição da prancheta para o ambiente digital. Passam a se constituir relações/interações menos diretas entre o arquiteto e a representação figurativa do objeto em concepção. Nascem as prerrogativas do DAD (Digital Architectural Design - Projeto Digital de Arquitetura): "distinguir a representação como o modo lógico e operativo dominante da geração formal no design" (OXMAN \& OXMAN, 2014, pag. xxvii, tradução dos autores). Em 2008 Rivka Oxman já refletia sobre as diferenças nos modos com os quais o arquiteto utilizava o ambiente digital:

Hoje há uma necessidade de fazer a distinção entre CAD (Computer-Aided Design) e DAD (Digital Architectural Design). A distinção entre CAD e DAD é muito mais do que simplesmente terminológica. Embora princípios, teorias e métodos de CAD (KALAY, 2004) tenham sido basicamente baseados na imitação de projetos baseados em papel, os novos conceitos de modelos de design digital estão reintroduzindo um meio diferente de conceituação, substituindo a mídia baseada em papel. (OXMAN, 2008, p.106, tradução autores)

Um denominador comum a estes autores é a ênfase conferida ao processo projetual no ambiente digital como um processo de pesquisa, possibilitando a visualização do Digital Architectural Design como meio de produção de conhecimento, o qual modifica radicalmente as práticas disciplinares. Compreendendo estes avanços, Oxman (2006) investiga a modificação no processo de projeto a partir da interação entre o arquiteto e a representação digital. Nesse artigo, a autora distinguiu três tipos de interações no ambiente digital, a partir da lógica da interação do projetista com papel. São eles: a) interaction with digital constructs (Interação com construções digitais) b) interaction with a digital representation generated by a mechanism (Interação com a representação digital gerada por um mecanismo) e c) interaction with digital environment that generates a digital representation (Interação com o ambiente digital que gera a representação digital).

As três interações descritas por Oxman (2006) foram posteriormente denominadas sinteticamente por Kotnik (2010) como "níveis de computabilidade". São eles, respectivamente: Representacional, Paramétrico e Algorítmico, como pode ser visualizado na sistematização apresentada na Figura 1.

O nível de computabilidade representacional, é aquele realizado a partir de um croqui, desenho ou modelo digital, cujo processo de projeto 
transpõe uma abordagem de design/projeto convencional em papel para o espaço digital. Para a melhor compreensão deste nível, pode-se assumi-lo como o da interação com uma prancheta eletrônica para representação bidimensional de elementos geométricos bi ou tridimensionais.

Pode-se compreender o nível de computabilidade paramétrico como uma representação digital gerada por regras pré-definidas. Neste nível, as resultantes formais podem ser diferenciadas dentro de um espectro de variações contínuas dos parâmetros. Todos os parâmetros são explícitos e vinculados segundo uma hierarquia de associações geométricas (BURRY, 2007). No momento em que o modelo é gerado, variações podem ser produzidas, transformadas e manipuladas pelo controle dos parâmetros, permitindo um espectro de possibilidades entre a entrada (input) e saída (output) de informações (KOTNIK, 2010).

Por sua vez, o nível de computabilidade algorítmico é caracterizado pela descrição formal gerada como uma estratégia de projeto/design, sendo desenvolvida por meio de operações algébricas, analíticas e geométricas que resultam em uma forma arquitetônica. A operação neste processo é realizada por meio de um sistema generativo, e seu desenvolvimento parte da concatenação destas operações, que emergem em uma forma arquitetônica. É importante destacar que as interações paramétrica e algorítmica se utilizam do desenho paramétrico, ou seja, devem existir parâmetros que sejam facilmente manipulados individualmente ou de maneira coordenada dependendo das relações pré-estabelecidas pelo designer, dando flexibilidade ao processo de projeto. Porém, o que difere as duas interações é que a segunda contém um processo generativo, no qual as variáveis iniciais não são diretamente proporcionais às variáveis finais (KOTNIK, 2010).

Tanto Oxman quanto Kotnik caracterizam os níveis de computabilidade paramétrico e algorítmico como "Digital Architectural Design", pois estabelecem-se por meio de uma utilização consciente do potencial dos elementos que definem uma função computável como ferramentas de design. A abordagem adotada no presente estudo, baseandose em Kotnik (2010), assume que existe uma ruptura entre o nível de computabilidade representacional e o nível de computabilidade Paramétrico, que demarca o limite do projeto digital - "Digital Design Threshold". A figura 1 apresenta a sistematização dos conceitos relativos às interações e as subcategorias de cada interação estruturados a partir de Oxman (2006), transpostos para os níveis de computabilidade de Kotnik (2010). 
Figura 1: Esquema - Níveis de interação Representacional, Paramétrico e Algorítmico

CAD (Computer Aided Design) / Digital Design Models (OXMAN, 2006)

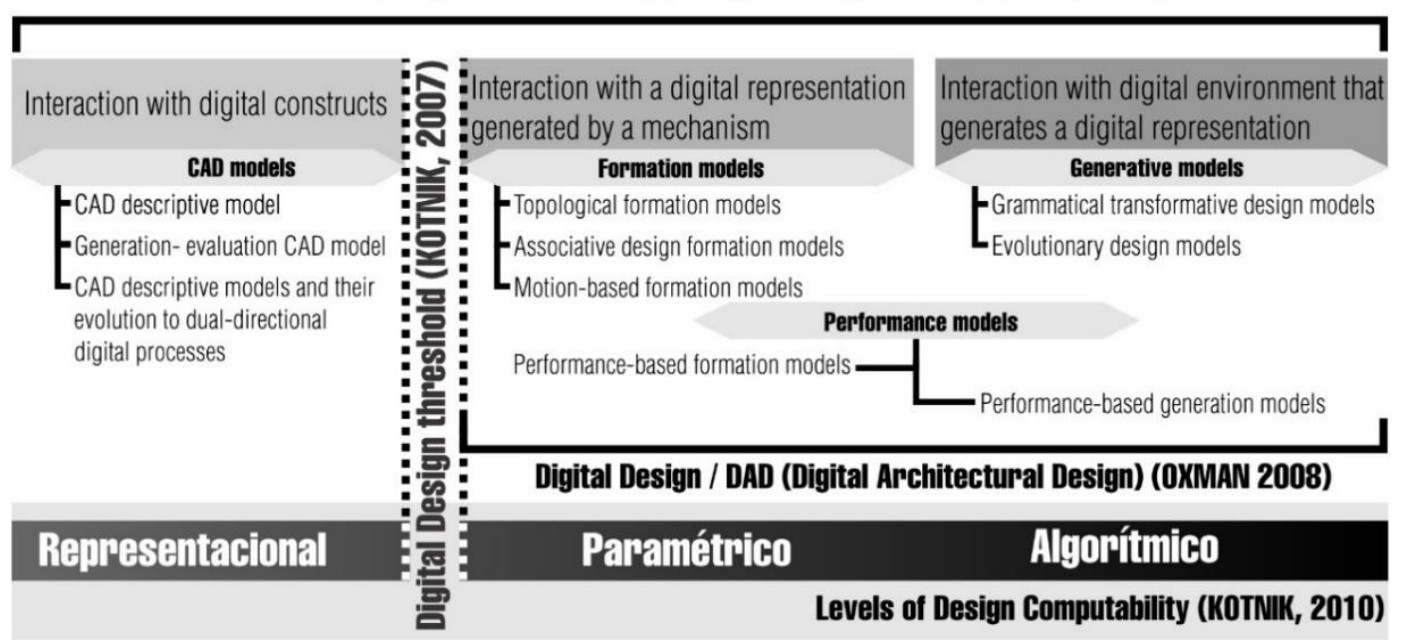

Fonte: Autores , baseado nas construções teóricas de Oxman (2006); Kotnik (2007); Oxman (2008) e Kotnik (2010).

É importante ressaltar que toda a discussão levantada aqui sobre os níveis de computabilidade tem como foco a compreensão dos modos de exploração das potencialidades da tecnologia digital, não implicando necessariamente na avaliação da qualidade ou performance dos projetos resultantes.

\subsection{Arquitetura, ensino e projeto digital: a importância de compreensão do contexto}

Diante, dos aspectos teóricos levantados até o momento, pretende-se com esta pesquisa suprir a inexistência de estudos que identifiquem e mapeiem estratégias didáticas nas escolas de Arquitetura segundo os tipos de interações ou níveis de computabilidade que estão sendo explorados. Compreendendo esta lacuna, esta investigação torna-se importante, visto que as estratégias projetuais exploradas têm significativa influência nas arquiteturas geradas.

Compreende-se, então, a importância da reflexão crítica sobre os métodos, ferramentas e práticas que vêm sendo desenvolvidas em âmbito acadêmico, assumindo o papel de educador e pesquisador preparado, como explicitado por Kalay (2009):

\footnotetext{
"As escolas de arquitetura, portanto, têm a responsabilidade de entender essas mudanças e de orientá-las, ao mesmo tempo em que educam as novas gerações de arquitetos que usarão esses métodos, ferramentas e práticas para mudar o ambiente em que vivemos. Esse é o nosso desafio, como educadores e pesquisadores: se não nos levantarmos para alcançá-lo, outros (como os fornecedores de ferramentas CAD) moldarão nossa disciplina e nossos ambientes por muitos anos." (KALAY, 2009, pag. 6, tradução nossa)
} 
Mas para que essa reflexão e as formas de atuação sejam situadas e realizadas a partir de um contexto específico e que a ele façam sentido, é oportuno compreender o real cenário de cursos de arquitetura e urbanismo no Brasil. Flório (2012) já apontou que no contexto brasileiro, em muitos cursos, as disciplinas de Informática mantinham um enfoque operacional e instrumental. Celani et. al, (2017) também afirmaram que as experimentações que utilizam das novas tecnologias distribuíam-se de maneira pulverizada pelo país e ainda eram experiências isoladas com foco no ensino de software.

Entre as observações que fizeram esses autores e o momento atual é preciso verificar se existiram alterações no cenário e, mais ainda, encontrar formas para inserção de processos que se utilizem de níveis de computabilidade mais avançados. Acreditamos que o primeiro passo para inserção de níveis de computabilidade avançados no ensino de projeto de arquitetura no Brasil é o mapeamento de dados quantitativos e qualitativos sobre o quadro do ensino de arquitetura no país em face às novas tecnologias digitais de projeto. Entende-se que a partir do conhecimento dessa realidade e de seu cruzamento com o panorama dos níveis de interação que vêm sendo relatados no ensino na graduação de arquitetura e urbanismo no Brasil, seria possível avançar futuramente em apontamentos para estratégias de introdução neste cenário de níveis mais avançados de computabilidade em processos de projeto.

\section{METODOLOGIA}

Neste artigo é apresentada parte de uma pesquisa em nível de doutorado em andamento acerca da apropriação de tecnologias de representação gráfica e design digital no ensino de arquitetura no contexto brasileiro. Esta pesquisa pretende apresentar um estudo descritivo (GIL, 2010) do contexto em questão, por meio de uma série de análises correlacionais (GROAT \& WANG, 2013). A pesquisa correlacional é focada em padrões de relacionamento entre duas ou mais variáveis de um contexto específico.

São utilizados dois conjuntos de dados, o primeiro relativo ao macro cenário dos cursos de arquitetura e urbanismo no Brasil, e o segundo conjunto referente a práticas que vem sendo desenvolvidas em ambientes digitais, que explorem processos projetivos. A leitura e os cruzamentos informacionais dos mapeamentos aqui propostos permitem a composição de um perfil. Entendese que é possível extrair certos perfis resultantes de informações institucionais e práticas de inserção de níveis de computabilidade mais avançados, os quais são dados obtidos a partir das classificações propostas na revisão sistemática.

\subsection{Mapeamento da Informação dos Cursos de Arquitetura e Urbanismo}

Compreende-se a potencialidade de uma investigação que se utilize da visualização de dados de um contexto específico, fazendo com que, conforme dizem Abrams and Hall (2006), o complexo torne-se acessível, e o invisível seja 
visível. Busca-se o mapeamento de informações como estratégia para dar visibilidade a um contexto, descortinando e apresentando relações por meio de dados concretos.

O mapeamento da informação sobre todos os cursos de graduação em Arquitetura e Urbanismo foi realizado de outubro de 2017 a fevereiro de 2018 por meio dos dados disponibilizados pelo Ministério da Educação e Cultura (MEC). Os dados foram extraídos manualmente e compilados em uma tabela específica para o estudo (Microsoft Excel 2017, Estados Unidos). As seguintes variáveis foram coletadas: Pontuação do curso de acordo com órgãos governamentais e exames nacionais; Número de locais autorizados; Modalidade e situação (sala de aula ou ensino a distância; aberto, em andamento, fechado); Implementação do curso (data); Natureza administrativa da instituição (privada ou pública); Cidade e estado.

É importante ressaltar que Maragno (2013) já realizou um estudo em relação aos dados quantitativos até o ano de 2013, indicando quantidade de cursos, relações entre cursos privados e públicos, e a quantidade de arquitetos por habitantes. Neste estudo, o autor apontou um crescimento expressivo da quantidade de cursos de graduação, totalizando 240 cursos até aquele ano.

\section{2 "Handsearching"}

Para desvendar as experimentações com os vários níveis de computabilidade no ensino de arquitetura e urbanismo no Brasil e consequentemente apresentar um panorama do contexto, utilizou-se como estratégia metodológica a revisão sistemática, a qual é estabelecida na literatura como transparente e passível de ser replicada (SAMPAIO e MANCINE, 2007).

Compreendendo como é desenvolvida a produção na área da arquitetura e urbanismo no Brasil, assumiu-se a pertinência de busca por artigos nos principais congressos da área específica. 0 escopo do presente artigo circunscreve-se à revisão sistemática de trabalhos publicados em anais do Graphica, tendo sido utilizada a classificação estabelecida por Kotnik (2010) apresentada anteriormente.

Devido à ausência de dados organizados e sistematizados em base de dados de congressos do Brasil, foi escolhida a estratégia da revisão sistemática por meio de handsearching (busca manual). Nas próximas etapas da pesquisa, os dados aqui relatados serão somados a outros que excedem o escopo deste artigo e que foram obtidos por revisão sistemática desenvolvida em Vasconselos e Sperling (2017), com a etapa de busca por palavras-chave na base de dados CuminCAD (Cumulative Index of Computer Aided Architectural Design).

O processo de handsearching foi dividido em 6 etapas. 


\subsubsection{Seleção da Base de dados}

No processo de identificação por meio de handsearching foram identificadas as publicações referentes aos anos de 2005, 2007, 2009, 2011, 2013, 2015 e 2017. A escolha do período justifica-se pela intenção de mapear o estado da arte no campo, paralelamente ao desenvolvimento e disponibilidade de software paramétricos como Generatives Components (2003), Grasshopper (2007) e Dynamo (2011), em resposta a questões abordadas em Herrera (2010) que apresentou uma predisposição de países da América Latina à utilização de ferramentas que exploram a linguagem de programação visual.

\subsubsection{Seleção de artigos}

No processo de seleção dos artigos inicialmente identificados na base de dados, procedeu-se a uma etapa de leitura de títulos e resumos. Os critérios para seleção foram:

-Práticas didáticas (atividades nas disciplinas, cursos experimentais e ou workshop) ou descrição de contexto; Urbanismo:

-Estudos realizados em cursos de graduação em Arquitetura e

-Estudos realizados em instituições brasileiras ou com parcerias com instituições estrangeiras:

-Atividades Guiadas: Compreende-se por estas atividades processos advindos de experimentações didáticas coletivas, excluindo desta amostra um processo individual. Pois sendo individual, poderia estar mais vinculada a um conhecimento de um curso externo à graduação, a um estágio ou intercâmbio; ligada portanto à trajetória constituída por um estudante especifico, e não ao processo de aprendizado que os alunos de determinada instituição vivenciaram coletivamente.

-Foco na escala do objeto e da arquitetura e exclusão da escala do urbanismo desta amostra;

-Atividades que envolvam a criação de um objeto, ou um processo projetual; consequente exclusão de propostas didáticas de "redesenho";

-Atividades utilizando-se do ambiente digital;

\subsubsection{Artigos incluídos}

A partir da amostra selecionada foi realizada uma leitura completa dos artigos, sendo incluídos apenas os que cumpriram especificamente os critérios anteriormente citados. É importante ressaltar que compreendemos como "propostas de redesenho" as atividades didáticas que replicam um desenho trazido pelo professor, focando-se na compreensão dos instrumentos.

\subsubsection{Classificação de artigos}

Com base no referencial teórico anteriormente abordado (OXMAN, 2006) sobre os avanços do processo de projeto estabelecidos em ambiente 
digital, e os níveis de computabilidade descritos anteriormente por Kotnik (2010), foi realizada a classificação dos processos digitais de projeto desenvolvidos nas práticas de ensino relatadas pelos artigos selecionados e incluídos na amostra, dividindo-os em: representacional, paramétrico e algorítmico. É importante ressaltar que toda a classificação foi feita a partir das descrições dos experimentos presentes nos artigos.

\subsubsection{Sistematização e mapeamento do cenário}

A partir da classificação dos trabalhos incluídos, foi realizada a descrição do estado da arte no que tange as questões de projeto auxiliados por computador. Em seguida, foi realizado o mapeamento temporal dos artigos frente à classificação.

\subsection{Correlação entre os dados}

No processo de cruzamento dos dados obtidos permitiu evidenciar, por exemplo, a relação de localizações geográficas com práticas didáticas e as variações dos níveis de computabilidade mais avançados, e ainda verificar estatisticamente suas correlações e influências. Esta camada de informação só é verificável com os quantitativos e mapeamento destas instituições. Foi possível ainda explorar estaticamente, se estas experiências se desenvolvem em grandes centros, em cursos mais ou menos recentes, em cursos privados ou públicos, dentre outras correlações.

A leitura e os cruzamentos de dados dos mapeamentos aqui propostos permitem a composição de um perfil, assim como pode-se também destinar o olhar para estratégias que fogem ao perfil dominante, e estudá-lo profundamente por meio de estudo de caso futuramente.

\section{DESENVOLVIMENTO}

\subsection{Mapeamento e visualização de dados como estratégia de imersão no contexto}

Diante dos dados sistematizados aqui, busca-se compreender em que momento e onde foram implantados os cursos de Arquitetura e Urbanismo presenciais, em atividade no Brasil. Neste momento, considera-se necessário desenvolver uma análise em duas escalas, a primeira com dados relativos ao contexto nacional, posteriormente aos contextos regionais. Foram levantados dados referentes à data de implementação dos cursos, dividindo o período analisado por décadas, conforme apresentado na Figura 2. É importante explicitar, que neste momento já temos uma tendência das próximas implementações, em cinza, as quais são instituições já cadastradas e aptas a iniciarem. 
Figura 2: Distribuição das escolas de Arquitetura e Urbanismo por data de início de atividade no Brasil, implementadas e não implementadas.

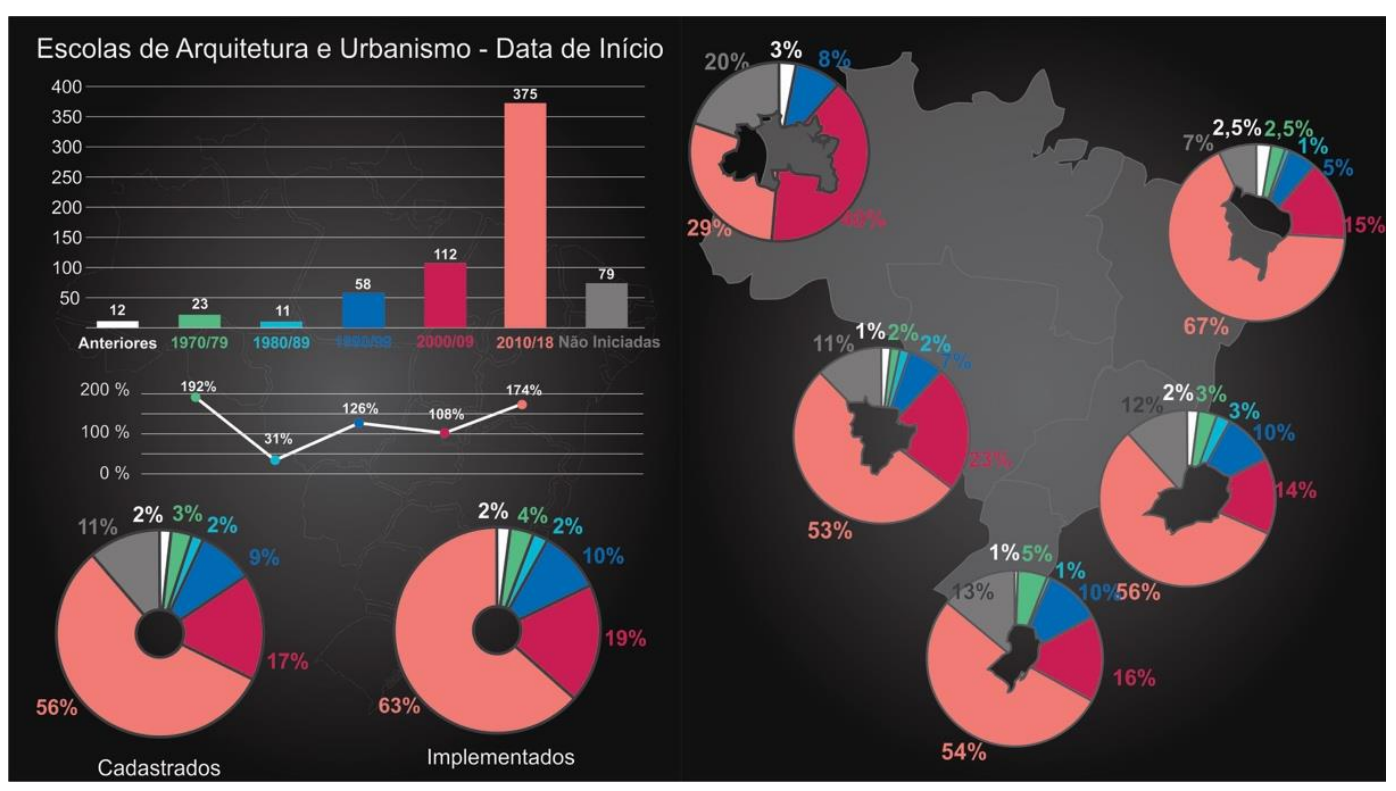

Fonte: Sistematização e visualização de dados desenvolvidas pelos autores a partir de dados extraídos da Plataforma do MEC, outubro de 2017 a fevereiro de 2018.

A partir dos gráficos da Figura 2, podem ser identificadas diferenças existentes na distribuição de cursos quanto a sua data de implantação, mas os dados tornam-se visualmente discrepantes se analisarmos a visualização da informação por mapeamento geográfico estadual de relações como quantidades de cursos, vagas disponíveis anualmente e a quantidade de vagas anuais relativas à quantidade de habitantes conforme apresentada na Figura 3.

Identifica-se, por meio da escala de cores, tanto na quantidade de cursos quanto a disponibilização de vagas, consolidando uma área que se espraia de Pernambuco até o Rio Grande Sul, caracterizando uma implementação maior em estados Litorâneos ou próximos ao litoral, no caso de Minas Gerais, que também está associado ao próprio histórico de desenvolvimento do país. Assim foi necessário analisar o cenário frente ao número de habitantes.

Destacam-se em posições opostas, como mostra o gráfico da direita da Figura 3, o Distrito Federal com aproximadamente 1 vaga anual disponível em curso de Arquitetura e Urbanismo para cada quase 900 habitantes, enquanto Roraima, tem uma vaga anual para aproximadamente 15.000 habitantes. 
Figura 3: Quantidade de cursos de arquitetura e urbanismo por estado (Esquerda); vagas disponíveis anualmente (centro); quantidade de vagas por habitantes por estado (direita).

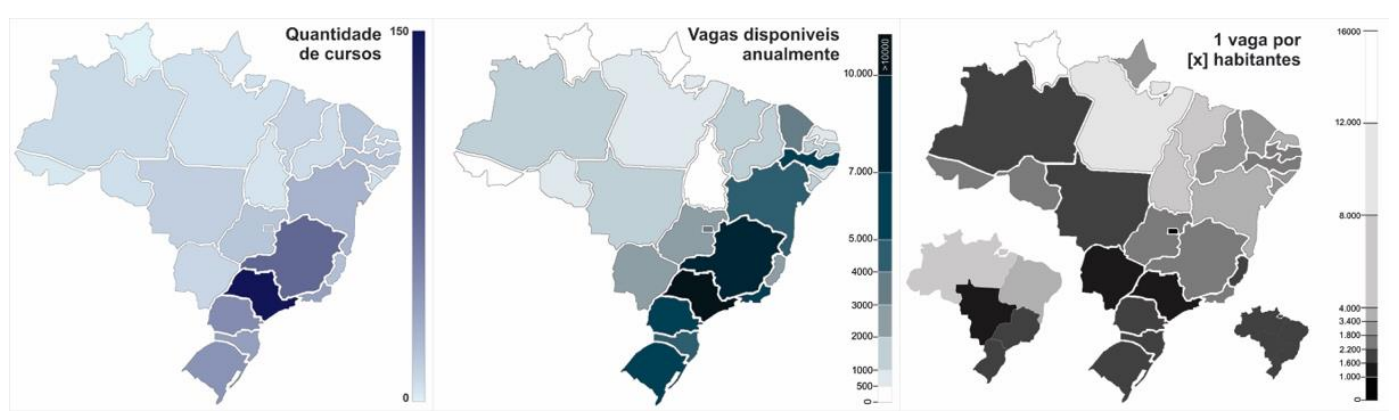

Fonte: Sistematização e visualização de dados desenvolvidas pelos autores a partir de dados extraídos da Plataforma do MEC, outubro de 2017 a fevereiro de 2018.

As diferenças permanecem evidentes nas relações entre quantidade de cursos privados versus cursos públicos (Figura 4 - esquerda), e ampliam-se se analisarmos o percentual de cursos implantados na capital e no interior.

Na relação entre instituições públicas e privadas, pode-se perceber que em grande maioria os estados apresentam uma maior quantidade de cursos privados, com uma única exceção (estado de Roraima - em cinza). A partir do mapeamento, percebe-se que uma maior quantidade de instituições privadas pode ser observada na Região Sudeste e Sul. Esta dinâmica avança significativamente no Centro Oeste e em parte do Nordeste.

Analisando a dinâmica de localização dos cursos por estados, relação de implantação na capital versus interior, acentuam-se as diferenças entre Norte e Sul. Pode-se identificar uma região contínua quase predominante na região sul e sudeste de cursos majoritariamente implantados em cidades do interior. Identifica-se igualmente o Centro-Oeste como uma região bastante heterogênea onde existe uma variação entre os estados, com quantitativos de $33 \%$ a $100 \%$ das instituições localizadas na capital 
Figura 4: Relações entre instituições públicas e privadas (esquerda); localização das instituições, situadas na capital ou no interior dos estados (direita).

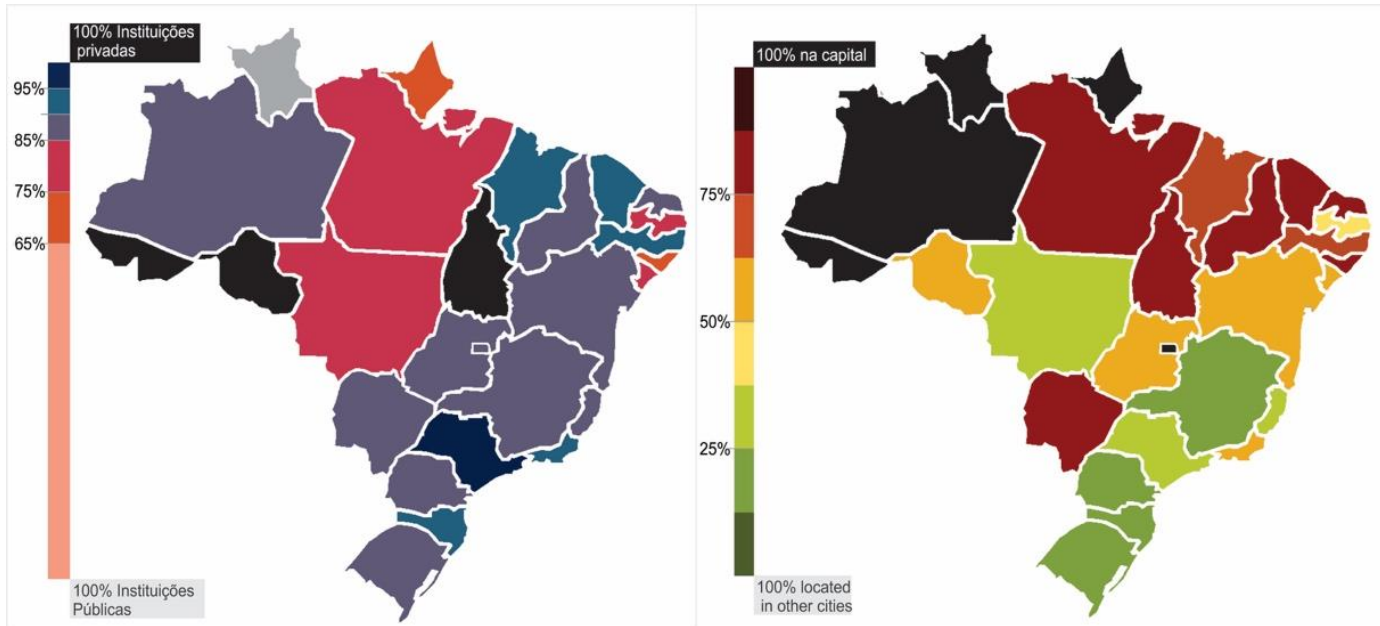

Fonte: Sistematização e visualização de dados desenvolvidas pelos autores a partir de dados extraídos da Plataforma do MEC, outubro de 2017 a fevereiro de 2018.

\subsection{Práticas didáticas}

No processo de construção da camada de informação referente às práticas didáticas em cursos de arquitetura e urbanismo e os níveis de interação segundo definidos aqui, parte-se de uma amostra de artigos apresentados em caráter específico e bienal no período de 2005-2017. Esta sistematização realizada por meio de handseraching, segue os mesmos passos de uma revisão sistemática. Os dados permanecem constantes nas três primeiras etapas, seleção da base de dados; identificação e elegibilidade, no caso 916 artigos, como pode ser visualizado na Figura 5, os quais são distribuídos em sete congressos, com uma variação entre as edições conforme o gráfico à direita da mesma figura. Esta lógica é estabelecida visto que não existe uma busca automatizada por palavras-chave e não se trata de uma base ampla onde apresentam-se artigos repetidos. Figura 5. 
Figura 5: Processo de sistematização do conteúdo sobre as práticas didáticas, da seleção da base de dados até a classificação dos artigos.

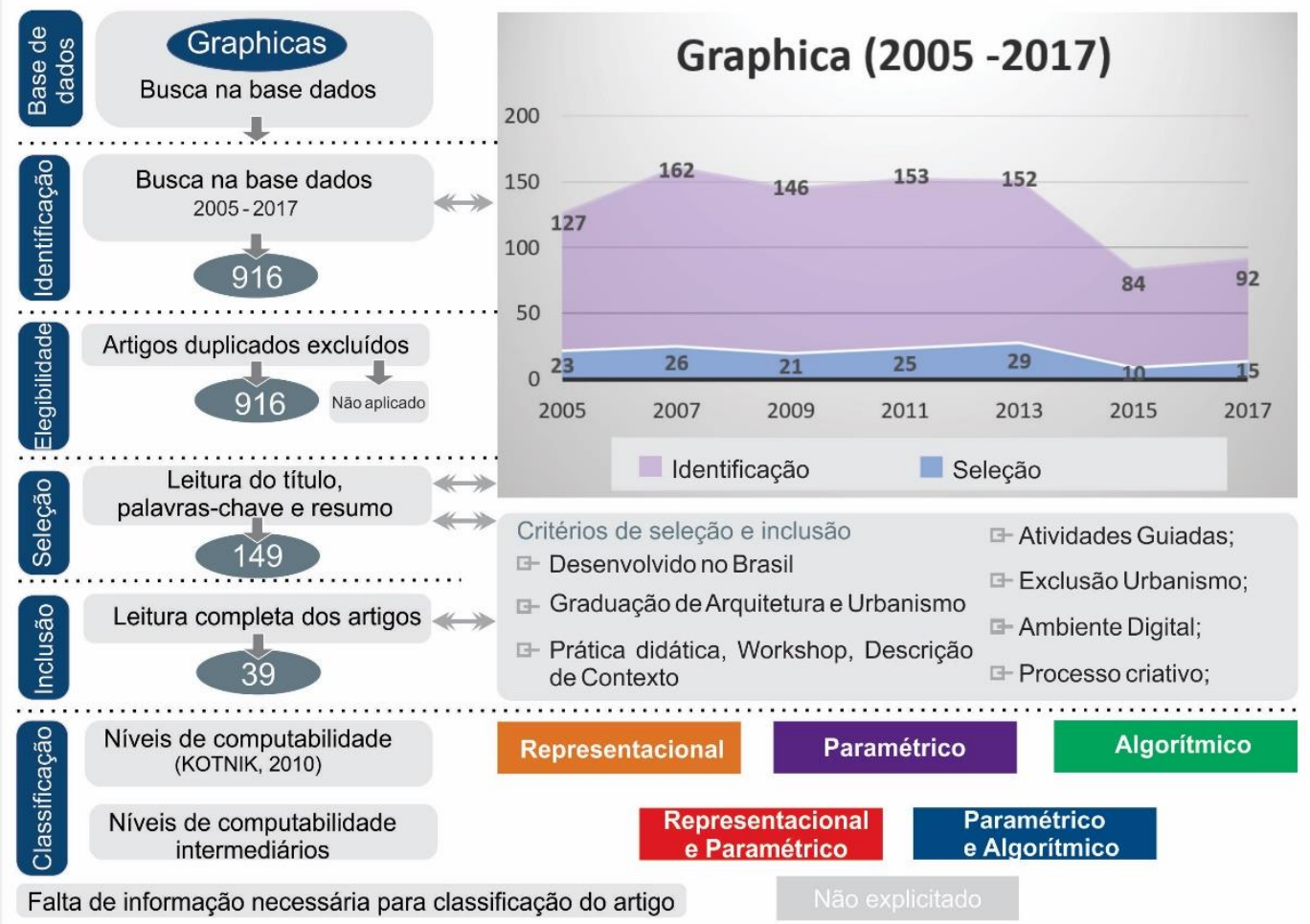

Fonte: Autores

Adotando os critérios explicitados durante o processo de seleção por meio da leitura dos títulos, palavras-chave e resumos, os artigos selecionados somados corresponderam a uma quantidade de 149 artigos, os quais neste momento seriam potencialmente relevantes para realização da classificação. $\mathrm{Na}$ continuidade, correspondente à fase de inclusão e referente à leitura completa dos artigos, a amostra finalizou em 39 artigos incluídos. É importante explicitar que a amostra excluída em sua maioria se tratava de trabalhos que não exploravam questões advindas do processo criativo, e sim tinham uma proposta de redesenho. Como um exemplo, o trabalho de autoria de Saugo (2013) que apresentou uma proposta focada na representação de um projeto utilizando-se das potencialidades do BIM (Building Information Modeling), e de um desenho já pré-estabelecido, sem abertura a alterações.

Experiências como essa, assim como outras que compunham os mais de 100 artigos não incluídos, tem um potencial de modificação do cenário em questão, no entanto, têm foco no aprendizado do instrumento e não na investigação projetual, não se enquadrando nos critérios de elegibilidade desta pesquisa. Ressalta-se, portanto, que diante do cenário atual estas experiências são importantes e necessárias, para uma modificação gradual.

Outras descrições de experiências foram excluídas pois se baseavam em processos criativos, mas não possuíam nenhuma interação no ambiente 
digital; ou tinham como "público alvo" profissionais de arquitetura ou estudantes de outras áreas.

É interessante ressaltar que diante de um dos critérios de inclusão, respectivo ao estudo ser desenvolvido em graduação no Brasil, houve exclusão de algumas experiências, como as realizadas em Cuba (González, 2009) e Portugal (Nogueira, et al. 2011). Estas são igualmente de extrema importância para fomentar a discussão do contexto e apresentar possibilidades aos pesquisadores, mas também não se enquadraram nos critérios de elegibilidade desta pesquisa.

Ainda, no processo de exclusão, destaca-se um trabalho com uma proposta em Python direcionada a um público de diferentes áreas do conhecimento, incluindo um curso de arquitetura. No entanto, como abordava diferentes cursos, o estudo teve que ser abrangente o bastante, e consequentemente o objetivo distanciou-se do que se compreende como um objeto arquitetônico, o que levou à exclusão do mesmo. Destaca-se que o mesmo seria bastante modificador do cenário por apresentar uma postura de investigação sobre aleatoriedade.

O processo de classificação inicialmente baseado em Oxman (2006) e Kotnik (2010), o qual indica três níveis de computabilidade nas experimentações foi ampliado conforme estudo prévio (VASCONSELOS; SPERLING, 2016; 2017) incluindo gradientes para análise de estudos que possuem mais de um nível de computabilidade neles presentes. A classificação estabelecida passou a incluir, então, os seguintes níveis de computabilidade: Representacional; Representacional e Paramétrico; Paramétrico; Paramétrico e Algorítmico e Algorítmico.

Compreendeu-se, ainda, que as interações denominadas de "intermediárias", foram realizadas a partir de diferentes estratégias didáticas, identificadas a seguir:

A. Estratégia de ensino com níveis de computabilidade independentes; artigos que apresentaram proposta didática que explora duas interações na mesma disciplina, porém como atividades independentes.

B. Estratégia de ensino com níveis de computabilidade mistos: artigos que apresentam atividades interdependentes, nas quais o processo de composição do modelo se estabelecia em cada momento com um tipo de interação.

C. Estratégia de ensino com níveis de computabilidade eletivos: artigos que apresentam atividades com interações a serem eleitas por cada participante no decorrer de seu processo projetual.

Ressalta-se ainda, que certos estudos não foram possíveis de serem classificados explicitamente em um ou mais níveis, tendo como exemplo Baldessin e Vizioli (2017), que aborda de maneira descritiva o contexto de projeto, dando indicações de possíveis utilizações de software, sem exatamente os identificar ou apresentar o processo de projeto explicitamente. 


\section{RESULTADOS E DISCUSSÕES}

Inicialmente identificam-se alguns cenários claros a partir dos dados correspondentes à classificação estabelecida, os quais estão apresentados na Figura 6, segundo porcentagens da amostra total e sua evolução temporal. Diante da amostra selecionada e de todo o processo de revisão sistemática realizado a partir dos critérios de seleção e inclusão, foi identificado que a maioria das práticas didáticas (80\%) partem de metodologias projetuais baseadas no nível de computabilidade representacional.

Reforçando este contexto brasileiro baseado em experimentações que se apropriam do computador transpondo o método analógico, é interessante perceber que as práticas que estão incorporando de alguma forma o Digital Architectural Design (10\%) baseadas no nível de computabilidade paramétrico, o fazem associando aos conhecimentos e práticas prévias, ou seja, o nível representacional (Figura 6).

Figura 6: Gráficos sobre as práticas identificadas.
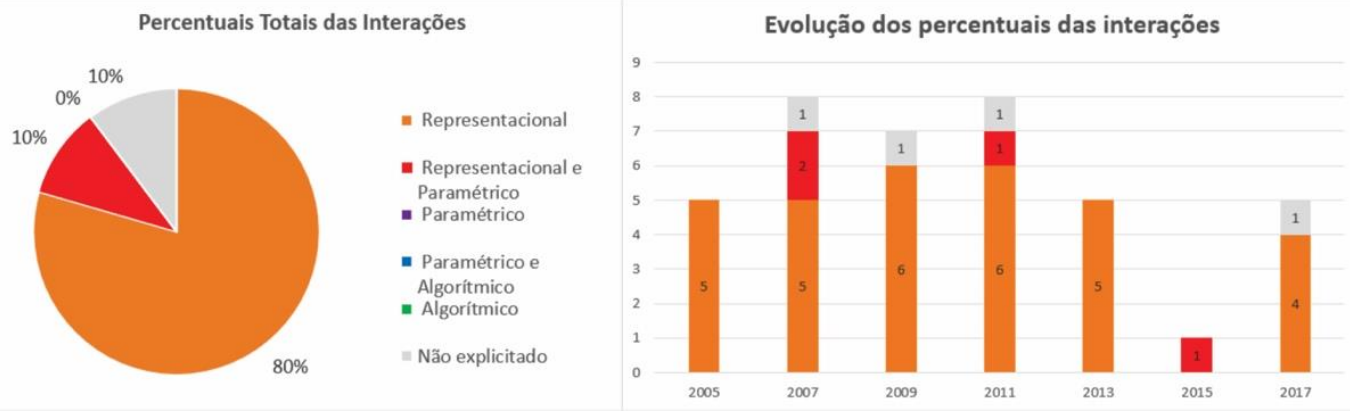

Fonte: Desenvolvido pelos autores.

As duas primeiras experimentações aparecem publicadas em 2007, posteriormente uma em 2011 e uma em 2015. É importante reforçar que estudos baseados em publicações sofrem um lapso temporal, entre a realização do estudo e a elaboração do artigo.

Deve-se destacar a pulverização e o número reduzido de implementações de DAD em estratégias didáticas presentes na amostra frente a outras amostras que recolhemos na pesquisa. Foi percebido ao longo do processo de inclusão dos artigos desta amostra o relato de precariedade de estrutura institucional. Autores abordam que já se tem o conhecimento e a vontade necessários para executar atividades no ambiente digital, até mesmo por parte dos alunos, havendo, no entanto, falta de recursos das instituições. Um exemplo é relatado por Franco (2005), sobre a existência de apenas um computador por sala. Nesta direção, existem os pesquisadores que tentam incluir alguma atividade no ambiente digital, porém devido à mesma falta de recursos, não poderiam torná-la obrigatória aos alunos sem apresentar o suporte necessário (PARAIZO e MATTOS, 2005). 
Podemos citar ainda experimentações na área que se aproximam e refletem de alguma forma sobre o DAD, no entanto estas experiências não estavam sendo aplicadas no âmbito do curso de graduação, diretamente estabelecidos em práticas em sala de aula (FLORIO; TAGLIARI, 2015; PEREIRA; VAZ, 2013), embora a última tenha indicado o interesse de aplicação na graduação no futuro.

Compreendemos ser importante destacar também o cenário sobre em que região do país e em que instituições estão sendo desenvolvidas as práticas no ambiente digital. Tem-se como resultado que $56 \%$ têm origem no Sudeste, $23 \%$ no Sul, e $21 \%$ no Nordeste, conforme apresenta a Figura 7. Por sua vez, as experimentações que se apoiaram no DAD foram desenvolvidas em três regiões, sendo duas no Sudeste, duas no Nordeste e uma no Sul.

Figura 7: Gráficos sobre as práticas identificadas.
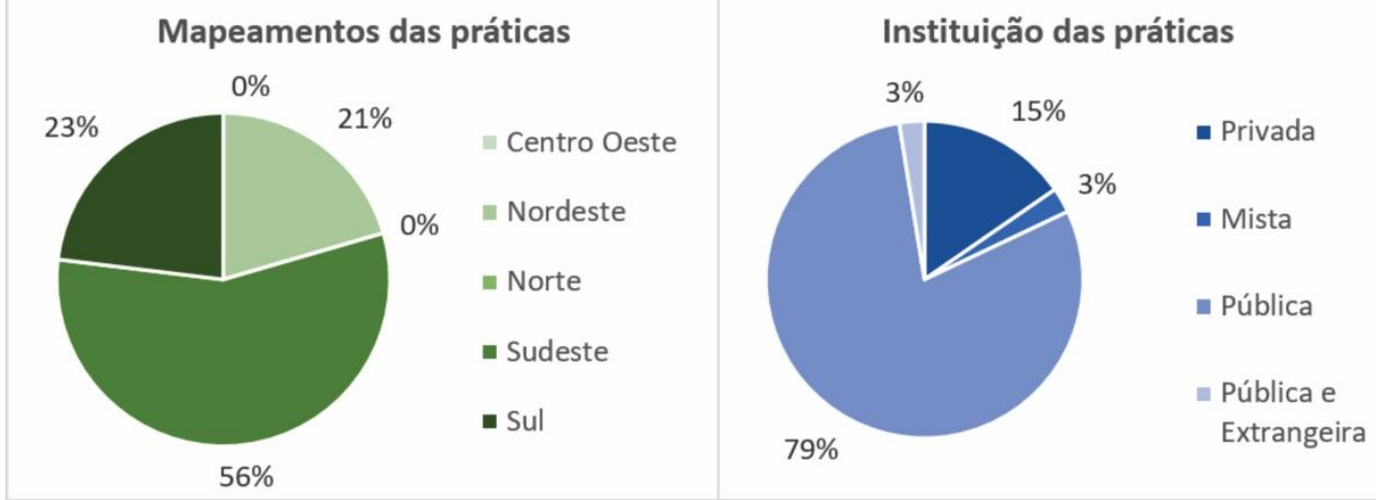

Fonte: Autores

O mapeamento sobre as instituições das práticas incluídas no estudo indica que $86 \%$ foram desenvolvidas em instituições públicas, sendo que deste montante um destes artigos (3\%) é correspondente a uma prática didática cuja exploração foi realizada conjuntamente com uma instituição estrangeira e outro correspondente a um trabalho desenvolvido conjuntamente a uma instituição privada. Assim os quantitativos referentes a instituições privadas são sobreposto a esta experiência conjunta, representando 18\%. Separando apenas incorporações de DAD, tem-se respectivamente $75 \%$ em instituições públicas e $25 \%$ em instituições privadas.

Quanto à localização destas explorações específicas, 50\% das práticas foram desenvolvidas no Nordeste, $25 \%$ sudeste e $25 \%$ no sul. Prevalências que não correspondem com a lógica da quantidade de curso em cada região, respectivamente $18 \%, 46 \%$ e $21 \%$. Este resultado, difere do cenário evidenciado no estudo prévio, o qual apresentava uma relação positiva entre a incidência de práticas didáticas com a quantidade de cursos por região. Ainda, 75\% das instituições com estas explorações distribuem-se nas capitais, enquanto que, ao analisarmos dados totais, apenas $40 \%$ dos cursos de Arquitetura e Urbanismo estão situados na capital. 


\section{CONSIDERAÇÕES FINAIS}

Inicialmente é interessante perceber que o contexto analisado é bastante conservador em relação à utilização da tecnologia, comparando-o aos dados apresentados em Vasconselos e Sperling (2017), o qual analisa um período semelhante com os mesmos critérios, em um contexto específico que tem como foco a exploração da gráfica digital. Acreditamos que essa questão é motivada pelo próprio foco do congresso Graphica e consequentemente do grupo de pesquisadores em questão, que valorizam e destacam a importância do desenho a mão.

Destaca-se que a intenção deste estudo não é diminuir ou invalidar métodos e técnicas já consolidadas na literatura, mas sim ampliar o leque de possibilidades segundo lógicas emergentes a partir da introdução da computação no processo projetual. Além da importância de apresentação deste cenário, espera-se motivar a exploração do ambiente digital no processo de projeto de arquitetura segundo os diversos níveis de computabilidade.

Reforça-se que o aprendizado de processos projetuais no ambiente didático deve estar aberto à pluralidades. Este estudo se interessou em captar possíveis transformações epistemológicas e das práticas diante do contexto das tecnologias digitais disponíveis para o projeto de arquitetura e a chegada de nativos digitais nas universidades. A partir da sistematização estabelecida e dos relatos presentes nos artigos selecionados, ainda que em número reduzido, vêm se mantendo presentes explorações do ambiente digital em processo de projeto com maior compreensão dos distintos níveis de computabilidade. Deve-se destacar que, em paralelo, estão em curso em instituições brasileiras muitas pesquisas direcionadas ao tema, tanto em nível de pós-graduação quanto de iniciação cientifica, as quais podem ter algum grau de repercussão nas experimentações realizadas no âmbito da graduação, mas que não foram captadas neste estudo.

\section{REFERÊNCIAS BIBLIOGRÁFICAS}

ABRAMS J and HALL P. Else/where: mapping. New cartographies of networks and territories. Minneapolis: University of Minnesota, 2006

BALDESSIN, Guilherme Quinilato; VIZIOLI, Simone Helena Tanoue. A aplicação de softwares de arquitetura e a utilização da linguagem visual nos trabalhos de graduação integrado.. In: Anais GRAPHICA 2017 - XII International Conference on Graphics Engineering for Arts and Design. Anais...Araçatuba(SP) UNIP, 2017.

BROADBENT, Geoffrey. Design in Architecture: Architecture and the Human Sciences. New York: John Wiley and Sons, 1973. 
BOTCHWAY, E.A; ABANYIE S.A.; AFRAM, S.O. The Impact of Computer Aided Architectural Design Tools on Architectural Design Education. The Case of KNUST. J Archit Eng Tech 4:145, 2015. doi:10.4172/2168-9717.1000145

BURRY JR. Mindful Spaces: Computational Geometry and the Conceptual Spaces in which Designers Operate. International Journal of Architectural Computing, 5, 4: 611-624, 2007.

CELANI , Gabriela. Cad criativo. Rio de Janeiro: Campos, 2003.

CELANI, Gabriela, Monteiro, A. M., Franco, J., \& Calixto, V. Integração de tecnologias CAD/CAE/CAM no ateliê de arquitetura: Uma aplicação no projeto de edifícios altos. Gestão \& Tecnologia De Projetos, 12(1), 29-52, 2017.

EASTMAN, Chuck; TEICHOLZ, Paul; SACKS, Rafael; LISTON, Kathleen . Manual de BIM: Um guia de modelagem da construção para arquitetos, engenheiros, gerentes, construtores e incorporadores. Porto Alegre: Bookman, 2014. 483 p.

FRANCO, Juarez Moara Santos. Modelos digitais no ensino de geometria descritiva potencialidades e limitações. In: GRAPHICA 2005. Anais. Recife, 2005.

FLORIO, W.; TAGLIARI, Ana . Modelagem e Simulação de Espaços Internos de Projetos Não-Construídos do Arquiteto Vilanova Artigas. In: GEOMETRIAS \& GRAPHICA 2015, 2015, Lisboa. Geometrias \& Graphica 2015: Tendências no Pensamento Gráfico: III Conferência Internacional da Aproged. XI International Conference on Graphics Engineering for Arts and Design. Lisboa: APROGED/Universidade Lusíada de Lisboa, 2015. v. 2. p. 123-135.

GIL, A. C. Como elaborar projetos de pesquisa. São Paulo: Atlas, 2002.

GONÇALES, Alexis Caridad Méndez. La expresión gráfica y lo digital. ¿esquemas conceptuales agotados? In: GRAPHICA 2009. Anais. São Paulo, 2009.

GROAT L and WANG D. Architectural Research Methods. 2th ed. Hoboken: John Wiley \& Sons, 2013.

HERRERA, Plabo. C. . Tecnologías disruptivas: programación y fabricación en Latinoamérica. SIGraDi 2010_Proceedings of the 14th Congress of the Iberoamerican Society of Digital Graphics. Bogotá, Colombia, November 1719, pp. 213-216, 2010.

KALAY, Yehuda. The impact of information technology on architectural design in the 21st century T. Tidafi and T. Dorta (eds) Joining Languages, Cultures and Visions: CAADFutures 2009, PUM, 2009, pp. 21-34 
KOLAREVIC, Branco. Architecture in the digital age: Design and manufacturing. Abingdom, Oxon: Taylor \& Frances, 2003.

KOTNIK, Toni. Algorithmic Architecture. In: MAS Colloquia 2006/07, p. 1-16. Disponível em: http://wiki.arch.ethz.ch/. Acesso em: setembro de 2018.

KOTNIK, Toni. Digital Architectural Design as Exploration of Computable Functions, in International Journal of Architectural Computing vol. 8 - no. 1, 1-16, 2010.

LAWSON, B. How designers think: the design process desmystified. Oxford: Architectural Press, 1980.

MARAGNO, G. V.. Quase 300 cursos de Arquitetura e Urbanismo no país: como tratar a qualidade com tanta quantidade? Algumas questões sobre qualificação e ensino no Brasil. Arquitextos, São Paulo, ano 14, n. 161.07, Vitruvius, out. 2013

<http://www.vitruvius.com.br/revistas/read/arquitextos/14.161/4930>.

MITCHELL, W. J. Beyond the Ivory Tower: constructing complexity in the digital age. Science Magazine, v. 303, n. 5663, mar. 2004.

OXMAN, Rivka. Theory and design in the first digital age. Design Studies 27. Londres: Elsevier, 2006.

OXMAN, R. "Digital architecture as a challenge for design pedagogy: theory, knowledge, models and medium". Design Studies. Great Britains: Elsevier, v 29 n 2 p.99-120, 2008.

OXMAN, Rivka; OXMAN, Robert. Theories of the Digital in Architecture. New York: ROUTLEDGE, 2014.

PARAIZO, Rodrigo Cury; MATTOS, Rodrigo Rinaldi de. Projeto urbano e representação por computação gráfica. In: GRAPHICA 2005. Anais. Recife, 2005.

PEREIRA, N. S. ; VAZ, Carlos E. V. . Parametrismo e ensino de geometria ? as superfícies de Félix Candela. In: GRAPHICA 2013, 2013, Floranópolis. Expressão Gráfica - Tecnologia e Arte para Inovação, 2013.

SAMPAIO R. F., MANCINI M. C. Estudos de revisão sistemática: um guia para síntese criteriosa da evidência científica. Rev Bras Fisioterapia. 2007;11(1):83-9.

SAUGO, Andréia. Autocad e revit architecture no ensino do desenho arquitetônico. In: GRAPHICA 2013. Anais. Florianopolis, 2013.

SILVA, Elvan. Uma introdução ao projeto arquitetônico. Porto Alegre: Ed. da Universidade, 1984. 
SILVA, Adriane Borda Almeida da; MARTINS, Gabriel; BRUM, Valentina Toaldo. A TRISSECÇÃO DO CUBO COMO LÓGICA EM AÇÕES PROJETUAIS DE ARQUITETURA.. In: Anais GRAPHICA 2017 - XII International Conference on Graphics Engineering for Arts and Design. Anais...Araçatuba(SP) UNIP, 2018.

VASCONSELOS, T. B. DE; SPERLING, D. M. . From representational to parametric and algorithmic interactions: A panorama of Digital Architectural Design teaching in Latin America. International Journal of Architectural Computing, vol: 15, issu 3, p. 215-229, 2017.

VASCONSELOS, T. B. DE; SPERLING, D. M. Entre representações, parâmetros e algoritmos: um panorama do ensino de projeto de arquitetura em ambiente digital na América Latina. In: XX Congreso de la Sociedad Iberoamericana de Gráfica Digital, 2016, Buenos Aires. Blucher Design Proceedings. São Paulo: Editora Blucher. p. 94-804.

WEBSTER, J. and WATSON, J.T.: Analyzing the past to prepare for the future: writing a literature review. MIS Quarterly \& The Society for Information Management, v.26, n.2, pp.13-23, 2002.

WOODBURY, R. Elements of Parametric Design. New York: Routledge, 2010.

\section{AGRADECIMENTOS}

0 presente trabalho foi realizado com apoio da Coordenação de Aperfeiçoamento de Pessoal de Nível Superior -Brasil (CAPES) - Código de Financiamento 001. Nesta direção, os autores agradecem as agências de financiamento que suportam esta investigação, em nível de doutorado anteriormente citada, e a bolsa de produtividade (CNPQ Processo 304071/2019-6), assim como ao Instituto de Arquitetura e Urbanismo da Universidade de São Paulo onde está sendo desenvolvida esta pesquisa. 